\title{
Fertility preservation for pediatric male cancer patients: illustrating contemporary and future options; a case report
}

\author{
Omar Abdelaal ${ }^{1,2 \#}$, Nicholas A. Deebel ${ }^{1,3 \#}$, Nima Pourhabibi Zarandi ${ }^{1 \#}$, Stanley Kogan ${ }^{1,3}$, \\ Frank C. Marini ${ }^{1}$, Thomas Pranikoff ${ }^{4}$, Kimberly Stogner-Underwood ${ }^{5}$, Thomas W. McLean ${ }^{6}$, \\ Anthony Atala ${ }^{1,3}$, Hooman Sadri-Ardekani ${ }^{1,3,5,6}$
}

${ }^{1}$ Wake Forest Institute for Regenerative Medicine, Wake Forest University School of Medicine, Winston-Salem, NC, USA; ${ }^{2}$ Department of Urology, Faculty of Medicine, Zagazig University, Zagazig, Egypt; ${ }^{3}$ Department of Urology, Wake Forest University School of Medicine, Winston-Salem, NC, USA; ${ }^{4}$ Section of Pediatric Surgery, Department of General Surgery, Wake Forest School of Medicine, Winston-Salem, NC, USA; ${ }^{5}$ Department of Pathology, Wake Forest University School of Medicine, Winston-Salem, NC, USA; ' ${ }^{6}$ ection of Pediatric Hematology/Oncology, Department of Pediatrics, Wake Forest School of Medicine, Winston-Salem, NC, USA

\#These authors contributed equally to this work.

Correspondence to: Hooman Sadri-Ardekani, MD, PhD. Wake Forest Institute for Regenerative Medicine, Section of Pediatric Hematology/Oncology, Department of Urology, Pathology, and Pediatrics, Wake Forest University School of Medicine, 391 Technology Way (WFIRM), Winston-Salem, NC 27101, USA. Email: hsadri@wakehealth.edu.

\begin{abstract}
The main aim of current pediatric male fertility preservation programs is storing spermatogonia stem cell (SSC) prior to starting cancer treatment. From July 1st, 2014 to May 1st, 2020; 170 patients have been recruited in Wake Forest Testicular Tissue Banking Program. The existence of multiple testis biopsies in different time points and detailed histological analyses of a unique cancer patient, provided an educational opportunity to investigate testis condition in different phases of cancer management. A pediatric male cancer patient with B-cell acute lymphoblastic leukemia (ALL) had multiple testicular leukemia recurrences and went through several testicular biopsies, to identify leukemic infiltration as well as considering fertility preservation. Infiltration of leukemia cells into both testes was identified. Neither elongated spermatid nor sperm were detected, but germ cells including SSC, spermatocyte and round spermatid could be identified in the stored tissue even after initial cancer treatment. Different germ cells were identified by hematoxylin and eosin (H\&E) staining and specific immunohistochemical (IHC) markers including PGP9.5/UCHL1 or MAGE-A4 (spermatogonia), SYCP3 (spermatocyte) and PRM1 (round spermatid). This emphasizes the importance of offering testicular biopsy to pediatric cancer patients at risk of infertility regardless to the stage of cancer treatment, although earlier biopsy is preferred. Promising research on in vitro spermatogenesis and auto-transplantation support the practice of SSC preservation. In addition, finding and storing round spermatids isolated from testicular biopsy provides a currently available option of round spermatid injection (ROSI). Given the complexity of managing cancer while considering fertility preservation, a multidisciplinary collaboration is important to achieve optimal overall outcomes.
\end{abstract}

Keywords: Male infertility; spermatogonia stem cell (SSC); fertility preservation; case report

Submitted May 03, 2020. Accepted for publication Oct 06, 2020.

doi: 10.21037/tau-20-908

View this article at: http://dx.doi.org/10.21037/tau-20-908

\section{Introduction}

Treatments for childhood cancer are often gonadotoxic and cause infertility up to $75 \%$ of male survivors (1). This adversely affects quality of life in adulthood (2). For post- pubertal males, semen banking is an effective method of fertility preservation. However, this is not the case for prepubertal children since they only have spermatogonia stem cells (SSC), the precursor cells for mature 
spermatozoa. As the cure rate of childhood cancer has increased to over $80 \%$ (3), the need for fertility preservation in prepubertal boys has been escalated (4).

A potential fertility preservation technique is to use stored SSC isolated from a testicular biopsy prior to cancer treatment. These cells could be cryopreserved and potentially transplanted back after cancer remission (5-7). While not yet demonstrated in humans, success has been achieved in multiple animal models including non-human primate (8). An important concern about this technique, especially in hematologic cancer patients such as leukemia is removing any malignant cells from the testicular biopsy to eliminate the risk of reintroducing cancer to the patient (9). It has been shown that germ cell transplantation with even minute contamination of leukemic cells could lead to transmission of leukemia (10).

We report a pediatric patient with $\mathrm{B}$-cell acute lymphoblastic leukemia (ALL) who participated in the testicular tissue banking program at Wake Forest Institute for Regenerative Medicine (WFIRM) (11) and describe the different analysis technique utilized to optimally determine fertility preservation options that could be applied to him based on recent state of the art technologies.

We present the following article in accordance with the CARE reporting checklist (available at http://dx.doi. org/10.21037/tau-20-908).

\section{Case presentation}

An 11-year-old Hispanic male was diagnosed with B-cell ALL. His genitourinary exam at original diagnosis was normal, with Tanner stage I development, and he did not undergo testicular biopsy at that time. He achieved remission with standard chemotherapy.

Three years later, while on maintenance chemotherapy (age 14), he developed fatigue and weight loss. A complete blood count showed pancytopenia, and a bone marrow aspirate showed recurrence of the ALL. At that time, he had Tanner stage III genitalia and bilateral, painless testicular enlargement, right greater than left. Ultrasound of the testicles confirmed the exam findings and was concerning for recurrent leukemia. The results of pathological examination of testis tissue at different biopsies are shown in Figure 1.

Under the same anesthesia for central line placement and a lumbar puncture, he underwent bilateral testicular biopsies for diagnostic purposes and oncofertility preservation. Testicular tissue showed the presence of germ cells including spermatogonia (Figure $1 A, B, D$ ), spermatocyte and round spermatid (Figure 1D). The numbers of the germ cells in the testes was much lower than in healthy age matched testis (Figure $1 M, N, P$ ). Hematoxylin and eosin (H\&E) and immunohistochemical (IHC) analysis also showed leukemic involvement in the interstitial tissue of the testes without invasion into seminiferous tubules (Figure 1C).

One year later, another testicular biopsy was performed due to further additional asymmetrical enlargement. The second biopsy was unilateral; no germ cells were present, and it was inconclusive for leukemia cells infiltration (Figure $1 E, F, G, H)$. The third biopsy was performed bilaterally 2 months later and confirmed extensive testicular leukemic infiltration and the absence of any germ cells (Figure 1I, $7, K, L$ ). Cerebrospinal fluid and bone marrow samples were both negative for ALL at that time.

Two weeks later, he then underwent bilateral orchiectomy. Pathology showed extensive involvement by B-cell lymphoblastic leukemia, and the complete absence of germ cells (data not shown). The timeline illustrating progress of the case is shown in Figure 2. No adverse event related to testes biopsies and orchiectomy were reported. Long term plan is "follow up with patient in andrology clinic for testosterone replacement therapy (TRT) and infertility treatment utilizing stored tissue including both SSC and round spermatid".

\section{Methods}

\section{Institutional Board Review (IRB) approval, and patient referral process}

Testicular Tissue Banking for Fertility Preservation in Boys and Men at Risk for Infertility (IRB00021686) was approved and launched in 2014 (11).

An automated alert was received from our electronic medical system (EMS) about a new oncology patient admission. This generated a referral from the Pediatric Oncology team to the Male Reproductive Urology (Andrology) and Regenerative Medicine teams. After consultation with the patient and his family, informed consent and assent forms were signed. Bilateral testicular biopsies were performed by the Surgery team under the same anesthesia utilized for a clinical indication such as central line placement. The testicular tissue was collected for spermatozoa identification, detailed histologic evaluation and cryopreservation (11). 


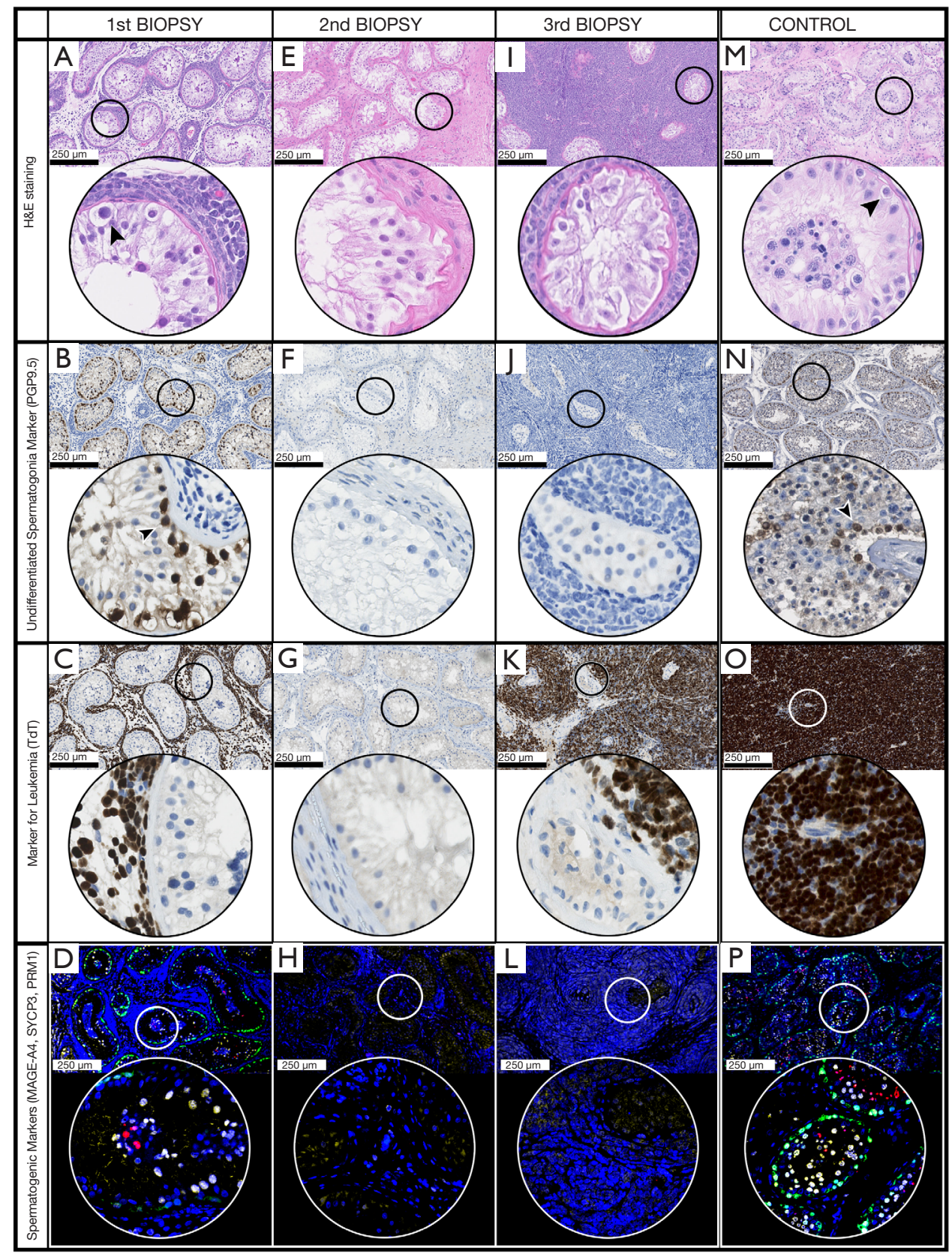

Figure 1 showing sections from different biopsies of the patient's testes. (A,B,C,D) First testis biopsy (at age 14, Tanner stage III) when the testicular tissue banking was done and it shows leukemic infiltration with some preserved spermatogonia, spermatocytes and round spermatids; (E,F,G,H) second testes biopsy 1 year later showed no spermatogonia and no leukemic infiltration; (I,J,K,L) third testes biopsy, showing dense leukemic infiltration and no spermatogonia. (A,E,I) Case report patient, (M) age matched control: H\&E staining; (B,F,J) case report patient, $(\mathrm{N})$ age matched control: PGP9.5 immunostaining showing spermatogonia in brown; (C,G,K) case report patient, (O) ALL bone marrow as positive control: TdT staining showing leukemic infiltration in brown; $(\mathrm{D}, \mathrm{H}, \mathrm{L})$ case report patient, $(\mathrm{P})$ age matched control: immunofluorescence staining for germ cell markers showing positive signal for MageA4 (green fluorochrome) marker for undifferentiated spermatogonia, SYCP3 (yellow fluorochrome) marker for spermatocyte, PRM1 (red fluorochrome) marker for round spermatid in the 1st biopsy but not in 2nd or 3rd, (P) positive control (age matched control). Blue fluorescent (DAPI, Nucleic acid marker). Arrow heads point spermatogonia cells in base membrane of seminiferous tubules. Scale bar 250 microns. H\&E, hematoxylin and eosin; ALL, acute lymphoblastic leukemia; TdT, terminal deoxynucleotidyl transferase. 


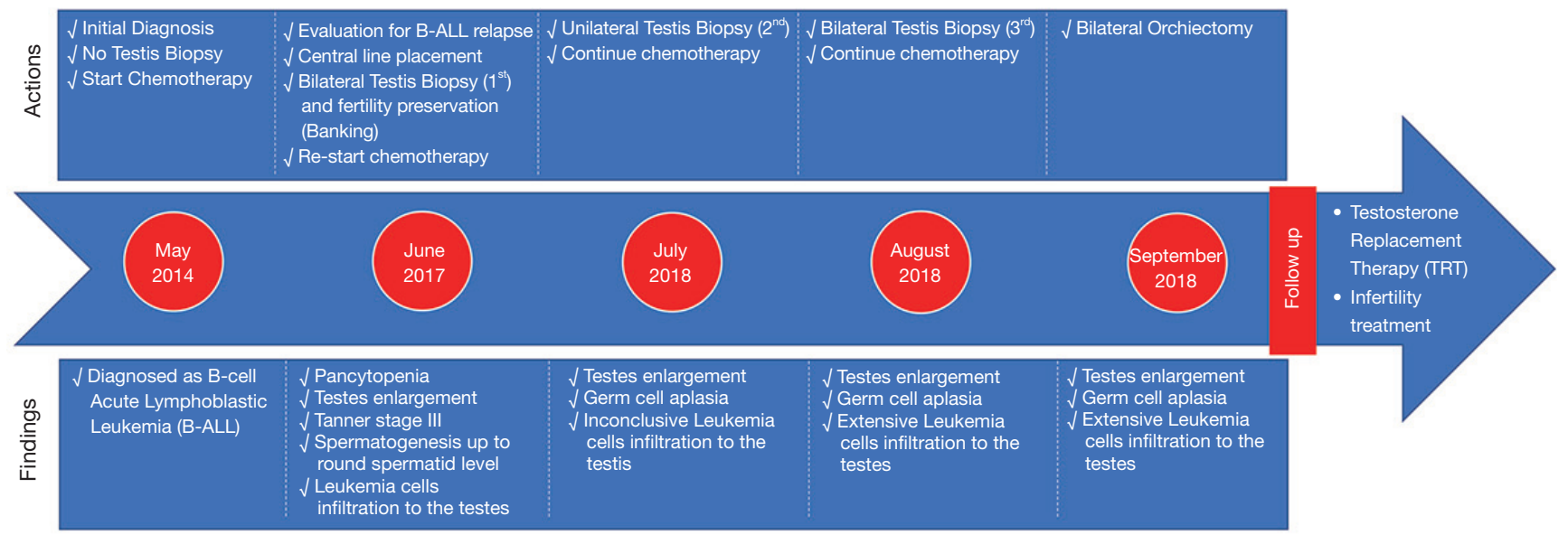

Figure 2 Illustrating case progression timeline and long term follow up.

\section{$H \& E$ staining}

To review the different types of testicular germ cells (i.e., spermatogonia, spermatocyte, round spermatid, elongated spermatid and spermatozoa) and the degree of leukemic infiltration of the testis, H\&E staining was performed on Bouin fixed tissue by using a Leica autostainer (Leica Biosystems Inc., ST5010 Autostainer XL). A dedicated urogenital pathologist reviewed all $\mathrm{H} \& \mathrm{E}$ slides to define the spermatogenesis level in preserved tissue, as well as to evaluate for presence of malignant cells.

\section{IHC staining to identify undifferentiated spermatogonia and leukemia cells}

IHC staining of PGP9.5 (UCHL1) an un-differentiated spermatogonia marker, and terminal deoxynucleotidyl transferase (TdT) a leukemia cells marker, were performed on Bouin fixed slides by using Bond 3 IHC Stainer from Leica Biosystems. Briefly, after 20 minutes of antigen retrieval using Bond Epitope Retrieval Solution 2 (ER2), the primary antibody (PA0286; mouse monoclonal PGP9.5 antibody or PA0339 mouse TdT, both from Leica Biosystems) was incubated for 15 minutes at room temperature (pre-diluted by company, ready to use). To detect and visualize the antigen, a Bond Polymer Refine Detection kit (contains a peroxide block, post primary, polymer reagent, DAB chromogen and hematoxylin counterstain) (Leica, DS9800) was used. Counterstaining with hematoxylin identified the nucleus of cells. Isotype control (mouse $\mathrm{IgG}$ ) was used as negative control instead of primary antibodies.

\section{Virtual microscopy}

Following H\&E and IHC staining, slides were scanned and analyzed using virtual microscopy technology under $40 \times$ magnification (NanoZoomer 2.OHT Digital Pathology, Hamamatsu Photonics).

\section{Immunofluorescence staining of germ cell markers}

Immunostaining was performed on paraformaldehyde (PFA) fixed slides using Opal 7-color IHC kit (NEL811001KT, PerkinElmer). After permeabilization of tissue using Triton $0.2 \%$ for 10 minutes, antigen retrieval was performed in AR6 buffer ( $\mathrm{pH} 6)$ at $95^{\circ} \mathrm{C}$ for 20 minutes. A PBS wash was performed three times (each time, 5 minutes) followed by incubation in a Dako protein block for 10 minutes at room temperature. Slides were incubated for 1 hour at room temperature sequentially in MageA4 mouse antihuman antibody (provided kindly as a gift from Prof. Giulio C. Spagnoli Basel/Switzerland) 1:50 dilution as an undifferentiated spermatogonia cell marker, SYCP3 antibody (Abcam ab15093) 1:3,000 dilution as spermatocyte cell marker and PRM1antibody (Sigma Aldrich 
HPA055150) 1:125 dilution as a round spermatid marker.

Opal polymer HRP mouse + rabbit was applied for 30 minutes followed by TBST wash. Different opal fluorochrome (10 minutes incubation at room temperature) was applied for each primary antibody, followed by TBST wash. Then antibody stripping (repeating antigen retrieval step) was performed prior to incubation of next primary antibody. After the last antibody staining was performed, spectral DAPI was applied for 5 minutes and washed by TBST. Slides were mounted using prolong gold antifade mounting medium (Invitrogen p36930) and gold seal cover glass (Thermo Fisher 3422ERI). Slides were then imaged using Olympus FV1200 laser scanning confocal microscope.

All procedures performed in studies involving human participants were in accordance with the ethical standards of the institutional and/or national research committee(s) and with the Helsinki Declaration (as revised in 2013). Written informed consent was obtained from the patient.

\section{Discussion}

Fertility preservation for pediatric cancer patients is an evolving field. We are reporting an example of a pre/ peripubertal cancer patient who would be eligible for and might benefit from participation in a fertility preservation program. This case also demonstrates the application of our current contemporary assessment techniques for evaluating pediatric fertility and preservation which might be used currently as well as future options for overcoming the lack of elongated spermatids or spermatozoa on biopsy $(6,7)$.

This patient was prepubertal (Tanner stage I) when he was originally diagnosed, therefore not yet able to ejaculate and produce spermatozoa for cryopreservation.

At the time of his original diagnosis (age 11), our testicular tissue banking program for fertility preservation was not yet available, however, he would have been a candidate to undergo a testicular biopsy prior to starting treatment. This way his SSC could be used in the future when stem cell treatments for infertility become available in humans. SSC transplantation has already been successful and led to production of spermatozoa in different species including mouse (12), rabbit (12) and monkey $(8,13)$ but has not yet been successfully accomplished in the humans.

This patient underwent three testicular biopsies when relapse was suspected. The first biopsy showed the presence of some spermatogonia, spermatocytes and round spermatids in his tubules after having gonadotoxic cancer therapy (Figure 1A,B,D). These findings suggest that banking should be offered at any timepoint in the patient's cancer therapy, even after previously treated with chemotherapy. In this case, given the total lack of germ cells on the third biopsy, it was very important to preserve the testicular tissue prior to the patient's need for a bilateral orchiectomy.

This patient had confirmed leukemic infiltration in his testes at the time of relapse (Figure 1C,K). One safe option for him would be to culture the SSC present in his testicular tissue to promote propagation $(14,15)$ then mature them in an organotypic culture $(16,17)$. This aims to achieve in-vitro spermatogenesis and spermatozoa production. Subsequently, these in vitro formed spermatozoa could be used for in vitro fertilization (IVF) to avoid the risk of reintroducing leukemia cells to the patient. While in vitro spermatogenesis has been successful in a variety of species, differentiation in the humans has only yielded round spermatids to date (18). Therefore, in vitro spermatogenesis is not yet an applicable clinical tool.

One of the patient's biopsies showed the presence of round spermatids in his seminiferous tubules (Figure 1D). Round spermatids can successfully be used for injection into activated oocytes, a technique known as round spermatid injection (ROSI). So far, this technique has resulted in over 90 births of healthy babies in Japan (19). Round spermatids could be extracted from cryopreserved testicular tissue to be used for ROSI. In order to bring the ROSI to routine clinical practice, we have recently started the first clinical trial (NCT04298255) in United Stated to test the safety and efficiency of ROSI (20).

The major limitation to this study was the unavailability of data about the state of the testis before starting chemotherapy and before leukemic infiltration. That could provide more information about the effect of chemotherapy on patient's testes, which would reflect upon our knowledge about the cumulative effect of gonadotoxic therapy.

\section{Conclusions}

This report demonstrates the use of contemporary fertility assessment and fertility preservation options for pediatrics male cancer patients. Options for fertility preservation should be tailored according to each case. We recommended consideration of testicular biopsy and SSC banking before starting gonadotoxic treatment, however if pretreatment banking cannot be achieved, i.e., before chemotherapy, banking can potentially still be performed. This should be done on a research protocol, as it is not yet a 
proven method. This patient still had some undifferentiated spermatogonia and post meiotic germ cells despite being treated with chemotherapy for 3 years. At relapse, this patient had significant leukemic infiltration in his testes. In order to avoid the risk of reintroducing the cancer to the patient after remission, in vitro spermatogenesis and IVF could potentially be the safest option for him when the technology becomes available. Additionally, given the presence of round spermatids on biopsy, ROSI could be a definite option. Options for fertility preservation should be tailored according to each patient's condition.

\section{Acknowledgments}

We thank Martha Ward, Kristine Ali, Brandi Bickford and Kenneth Grant (Wake Forest diagnostic pathology laboratory and imaging core) for their technical assistance on slide preparation, automated immunohistochemistry, virtual microscopy and laser scanning imaging. We thank Kristina Stumpf and Lysette A. Mutkus for their technical advices on Opal immunofluorescence staining.

Funding: Wake Forest Institute for Regenerative Medicine (WFIRM) internal funding.

\section{Footnote}

Reporting Checklist: The authors have completed the CARE reporting checklist. Available at http://dx.doi.org/10.21037/ tau-20-908

Conflicts of Interest: All authors have completed the ICMJE uniform disclosure form (available at http://dx.doi. org/10.21037/tau-20-908). The authors have no conflicts of interest to declare.

Ethical Statement: The authors are accountable for all aspects of the work in ensuring that questions related to the accuracy or integrity of any part of the work are appropriately investigated and resolved. All procedures performed in studies involving human participants were in accordance with the ethical standards of the institutional and/or national research committee(s) and with the Helsinki Declaration (as revised in 2013). Written informed consent was obtained from the patient.

Open Access Statement: This is an Open Access article distributed in accordance with the Creative Commons Attribution-NonCommercial-NoDerivs 4.0 International
License (CC BY-NC-ND 4.0), which permits the noncommercial replication and distribution of the article with the strict proviso that no changes or edits are made and the original work is properly cited (including links to both the formal publication through the relevant DOI and the license). See: https://creativecommons.org/licenses/by-nc-nd/4.0/.

\section{References}

1. Wallace WH, Anderson RA, Irvine DS. Fertility preservation for young patients with cancer: who is at risk and what can be offered? Lancet Oncol 2005;6:209-18.

2. Armuand GM, Wettergren L, Rodriguez-Wallberg KA, et al. Desire for children, difficulties achieving a pregnancy, and infertility distress 3 to 7 years after cancer diagnosis. Support Care Cancer 2014;22:2805-12.

3. SEER Cancer Statistics Review (CSR) 1975-2016. 2020. Available online: https://seer.cancer.gov/csr/1975_2016/

4. Sadri-Ardekani H, Akhondi MM, Vossough P, et al. Parental attitudes toward fertility preservation in boys with cancer: context of different risk levels of infertility and success rates of fertility restoration. Fertil Steril 2013;99:796-802.

5. Zarandi NP, Galdon G, Kogan S, et al. Cryostorage of immature and mature human testis tissue to preserve spermatogonial stem cells (SSCs): a systematic review of current experiences toward clinical applications. Stem Cells Cloning 2018;11:23-38.

6. Sadri-Ardekani H, Atala A. Testicular tissue cryopreservation and spermatogonial stem cell transplantation to restore fertility: from bench to bedside. Stem Cell Res Ther 2014;5:68.

7. Sadri-Ardekani H, Atala A. Regenerative medicine for the treatment of reproductive system disorders: current and potential options. Adv Drug Deliv Rev 2015;82-83:145-52.

8. Fayomi AP, Peters K, Sukhwani M, et al. Autologous grafting of cryopreserved prepubertal rhesus testis produces sperm and offspring. Science 2019;363:1314-9.

9. Abdelaal $\mathrm{O}$, Barber $\mathrm{H}$, Atala A, et al. Purging of malignant cell contamination prior to spermatogonia stem cell autotransplantation to preserve fertility: progress \& prospects. Curr Opin Endocrinol Diabetes Obes 2019;26:166-74.

10. Jahnukainen $\mathrm{K}$, Hou M, Petersen C, et al. Intratesticular transplantation of testicular cells from leukemic rats causes transmission of leukemia. Cancer Res 2001;61:706-10.

11. Sadri-Ardekani H, McLean TW, Kogan S, et al. Experimental testicular tissue banking to generate 
spermatogenesis in the future: a multidisciplinary team approach. Methods 2016;99:120-7.

12. Shinohara T, Inoue K, Ogonuki N, et al. Birth of offspring following transplantation of cryopreserved immature testicular pieces and in-vitro microinsemination. Hum Reprod 2002;17:3039-45.

13. Liu Z, Nie YH, Zhang CC, et al. Generation of macaques with sperm derived from juvenile monkey testicular xenografts. Cell Res 2016;26:139-42.

14. Sadri-Ardekani H, Mizrak SC, van Daalen SK, et al. Propagation of human spermatogonial stem cells in vitro. JAMA 2009;302:2127-34.

15. Sadri-Ardekani H, Akhondi MA, van der Veen F, et al. In vitro propagation of human prepubertal spermatogonial stem cells. JAMA 2011;305:2416-8.

16. Pendergraft SS, Sadri-Ardekani H, Atala A, et al. Threedimensional testicular organoid: a novel tool for the study

Cite this article as: Abdelaal $\mathrm{O}$, Deebel NA, Zarandi NP, Kogan S, Marini FC, Pranikoff T, Stogner-Underwood K, McLean TW, Atala A, Sadri-Ardekani H. Fertility preservation for pediatric male cancer patients: illustrating contemporary and future options; a case report. Transl Androl Urol 2021;10(1):520526. doi: 10.21037/tau-20-908 of human spermatogenesis and gonadotoxicity in vitro. Biol Reprod 2017;96:720-32.

17. Sato T, Katagiri K, Kubota Y, et al. In vitro sperm production from mouse spermatogonial stem cell lines using an organ culture method. Nat Protoc 2013;8:2098-104.

18. Galdon G, Atala A, Sadri-Ardekani H. In vitro spermatogenesis: how far from clinical application? Curr Urol Rep 2016;17:49.

19. Tanaka A, Suzuki K, Nagayoshi M, et al. Ninety babies born after round spermatid injection into oocytes: survey of their development from fertilization to 2 years of age. Fertil Steril 2018;110:443-51.

20. Experimental round spermatid injection (ROSI) to treat infertile couples (ROSI). 2020. Available online: https:// clinicaltrials.gov/ct2/show/NCT04298255?cond=ROSI\&d raw $=2 \& \operatorname{rank}=1$ 